Mini Review

\title{
Research Advance of Multi-anionic Compound Nanomaterials in Electrocatalytic Water Decomposition
}

\author{
Xin Liu ${ }^{1,2}$, Lin Zhu' ${ }^{2}$ Keying Cui ${ }^{1,2}$, Runyu Gao ${ }^{2}$,Yan Liu $^{2}$, Jialin $X u^{2}$, Haoran Ma ${ }^{2}$, and Weimin Du ${ }^{2, *}$ \\ ${ }^{1}$ College of Chemistry, Zhengzhou University, Zhengzhou, Henan, 450001, China \\ ${ }^{2}$ Henan Province Engineering Laboratory of Chemical Energy-saving Material Development and \\ Application, College of Chemistry and Chemical Engineering, Anyang Normal University, Anyang, \\ Henan, 455002, China, \\ *E-mail: dwmchem@163.com
}

doi: $10.20964 / 2021.07 .14$

Received: 1 March 2021 / Accepted: 7 April 2021 / Published: 31 May 2021

Due to the large surface area, lots of active sites and the better synergy, multi-anionic compound nanomaterials usually have the improved electrocatalytic activity and other special physicochemical properties. However, compared to the synthesis and catalytic application of multi-cation compound nanomaterials, the research on multi-anionic compound nanomaterials is lagged a lot, especially in the field of hydrogen production by electrolysis of water. Therefore, the recent research advance of multianionic compound nanomaterials are detailed reviewed in this paper, including: preparation methods, structural characteristics, electrocatalytic performance and internal mechanism. Some suggestions are made for the future development of multi-anion compound nanomaterials.

Keywords: Research advance; Multi-anionic; Nanomaterials; Electrocatalysis; Water decomposition.

\section{$\underline{\text { FULL TEXT }}$}

(C) 2021 The Authors. Published by ESG (www.electrochemsci.org). This article is an open access article distributed under the terms and conditions of the Creative Commons Attribution license (http://creativecommons.org/licenses/by/4.0/). 